\title{
MCNP calculations of neutron emission anisotropy caused by the GIT-I2 hardware
}

\author{
Ondřej Šíla, \\ Daniel Klír, \\ Karel Rezáč, \\ Balzhima Cikhardtova, \\ Jakub Cikhardt
}

\begin{abstract}
The MCNP6 and MCNPX calculations for the GIT-12 device in Tomsk were performed to determine the influence of the gas-puff hardware on the neutron emission anisotropy and the neutron scattering rate. A monoenergetic $2.45 \mathrm{MeV}$ neutron source and F1 and F6 tallies were declared in the simulation input. A comparison between MCNP results and the measured data was made. Differences between MCNPX and MCNP6 output data were investigated. In the experiment, two nTOF scintillation detectors with the Bicron BC-408 scintillator were used to measure the neutron waveform. Four bubble BD-PND detectors were used to estimate the amount of neutrons in different places around the neutron source.
\end{abstract}

Key words: Z-pinch • gas-puff $\bullet$ MCNP

O. Šíla ${ }^{\bowtie}$, D. Klír, K. Řezáč, B. Cikhardtova, J. Cikhardt Faculty of Electrical Engineering,

Czech Technical University,

Technicka 2, 16627 Prague 6, Czech Republic, Tel.: +420 22435 2359, Fax: +42023333 7031,

E-mail: ondrej.sila@fel.cvut.cz

Received: 11 June 2014

Accepted: 23 January 2015

\section{Introduction}

At present, there is a growing interest in pulsed fast neutron sources which may be useful in various applications like radiobiology, nuclear medicine or radiation material science [1]. Since there is need for a repetitive neutron sources, the deuterium Z-pinch became a more remarkable subject of research than in the past, when it mainly served as an effective source of X-rays [2]. Apart from the greatest $\mathrm{Z}$-pinch device - the $\mathrm{Z}$ machine with the neutron yield $4 \times 10^{13}$ [3], there are several smaller but still quite effective deuterium gas-puff devices producing relatively large amounts of fast neutrons. One of them is the GIT-12 device, situated in Tomsk, Russia, which is able to produce more than $10^{12}$ neutrons per shot.

For neutron diagnostic purposes, the information about the amount of scattered neutrons at the location of the detector is of considerable importance. Such value is not easy to determine in experimental way, thus the MCNP program was chosen to estimate this quantity.

\section{Experimental arrangement and simulation parameters}

GIT-12 is a current generator working under a microsecond implosion regime, with an intermediate inductive storage of energy and a microsecond plasma opening switch [4]. At $50 \mathrm{kV}$ of Marx charge voltage, it provides a current of 4.7 MA with the rise time of $1.7 \mu \mathrm{s}$ in short-circuit load [5]. 
In GIT-12 measurements, personal neutron dosimeters provided the determination of neutron yield. The bubble detectors are presently the most accurate means of neutron dose estimation and they are sensitive neither to dose rate nor energy, which makes them an ideal tool for neutron yield measurements. The presented data come from experiments with the BD-PND detectors which were placed at four various angles and distances around the americium-beryllium source, emitting $10^{6}$ neutrons/s. The detectors were irradiated for 62 hours. Due to this time scale, the total dose radiated to $4 \pi$ solid angle might be regarded as isotropic.

In the MCNP calculations, a monoenergetic isotropic point neutron source of $2.45 \mathrm{MeV}$ neutrons (the mean energy of the neutron of D-D fusion) was used. The F6 (the waveform of deposited energy in a specific cell) and F1 (the amount of neutrons crossing a selected surface) tallies were declared in the simulation input to obtain the needed quantities. Figure 1 and Fig. 2 illustrate the parts of the GIT-12 geometry which were implemented into the simulation.

\section{Results}

\section{MCNPX simulation of the waveform in the axial probe}

The simulation of the waveform of the deposited neutron energy in the scintillator material was executed for an axial detector located $10.12 \mathrm{~m}$ from the neutron source. The processes between the interaction of

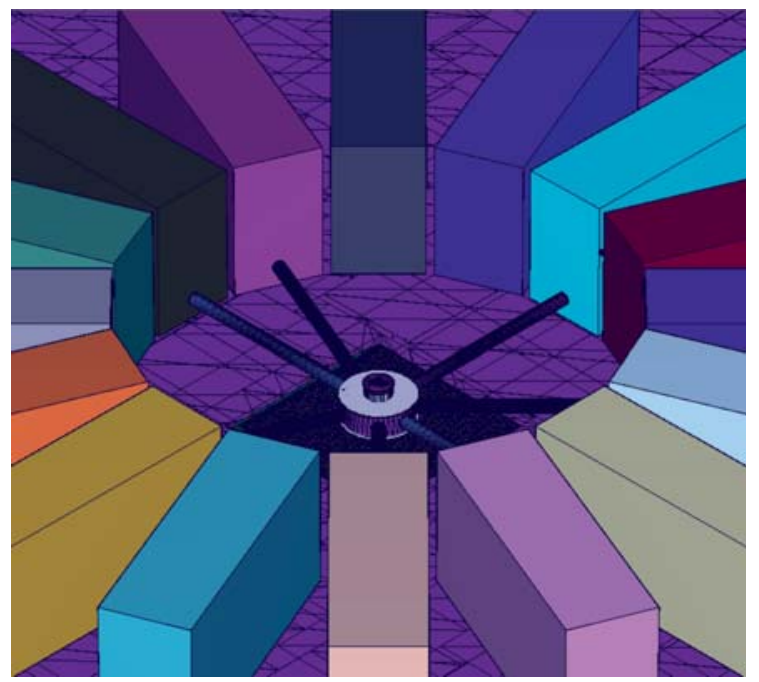

Fig. 1. A 3D view of the MCNP model of GIT-12.

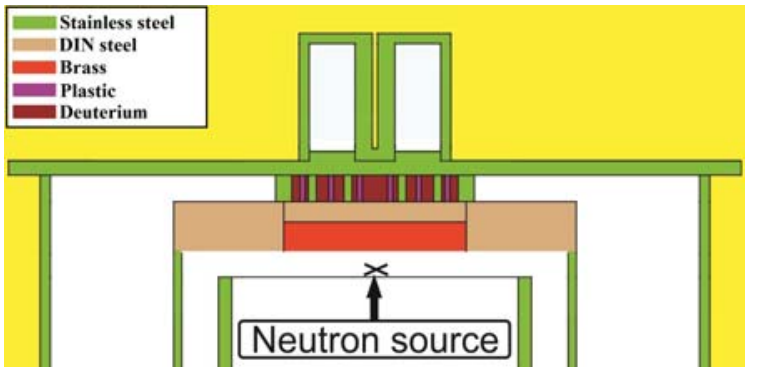

Fig. 2. The implemented parts of the GIT-12 gas-puff system.

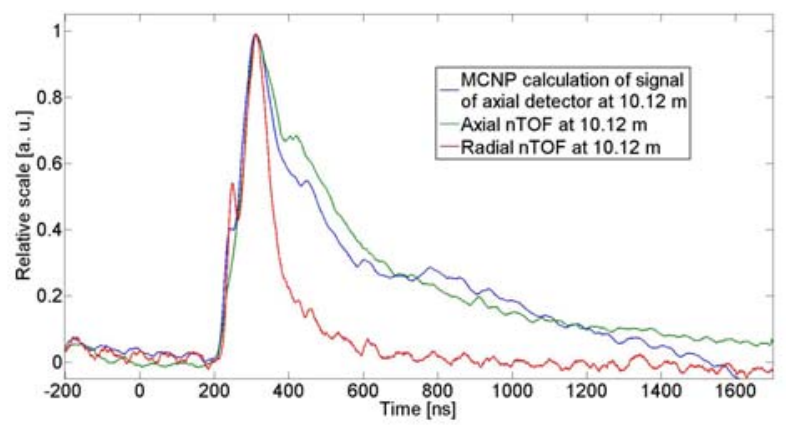

Fig. 3. The waveform of the radial and the axial probes (experimental TOF signals) and the MCNPX calculation of the waveform of the axial detector.

neutron with the scintillator and the output signal of detector were not included in the computer calculation (the contributions to the tally F6 time bins are scored immediately after interactions between the neutron and the matter), thus, before a comparison with the experimental data, the MCNPX result was convoluted with the signal of the radial probe, which is considered to be unaffected by neutron scattering $[1,6]$. Figure 3 shows the computed waveform and both the radial and the axial signals from the detectors.

The MCNP waveform is of very similar shape as the measured axial signal, hence there is probably a significant correlation between the computer simulation and reality.

\section{Influence of the GIT- I 2 hardware on the anisotropy} of the neutron emission

The analysis of the influence of the GIT-12 hardware on the neutron anisotropy, a crucial piece of information which is needed for most of neutron measurements, was performed by a MCNP6 simulation where additional annular surfaces were implemented into the GIT-12 geometry and uniformly distributed around the neutron source [7]. The total amount of neutrons crossing each surface was observed. Figure 4 describes this situation.

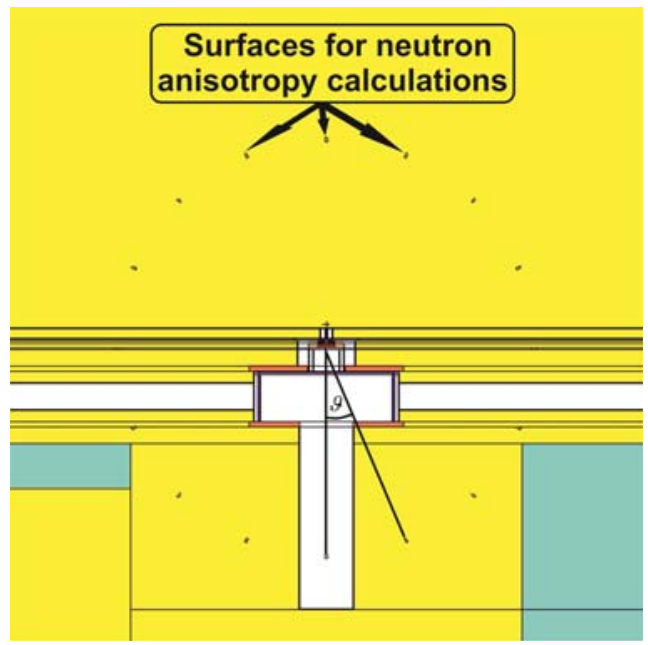

Fig. 4. The position of 16 surfaces $\left(20 \mathrm{~cm}^{2}\right.$ each) located at the distance of $2 \mathrm{~m}$ from the neutron source (one of the three considered distances). The angle $\vartheta$ represents the polar angle in Figs. 5, 6 and 7. 
The tally F1 simulation result, which estimated neutron dose in the 48 surfaces (16 surfaces belonged to each of the three distances that are $0.9 \mathrm{~m}$, $2 \mathrm{~m}$ and $4 \mathrm{~m}$ from the neutron source) is presented in Fig. 5. The amount of neutrons crossing the surface in $0.9 \mathrm{~m}$ at angle of $90^{\circ}$ was chosen as the reference number, and all the data (both calculated and experimental) were related to this value (measured quantities were divided by the PND value of $0.9 \mathrm{~m}$ and $\vartheta=90^{\circ}$ ). It should be noted that the number of the experimental results is quite low due to insufficient amount of the measured data.

The rate of scattered neutrons in a specific place is also of interest. It may be determined by the value of $\mathrm{N} / \mathrm{N}_{0}$, where $\mathrm{N}$ is the total number of neutrons crossing the surface, and $\mathrm{N}_{0}$ represents the un-scattered fraction contained in all $\mathrm{N}$ particles. The value of $\mathrm{N}_{0}$ was obtained from a simulation in which all the geometry was removed, so there were no scattering elements between the source and the place of detection. The angular dependence of $\mathrm{N} / \mathrm{N}_{0}$ value is shown in Fig. 6.

In directions represented by $\vartheta$ greater than $90^{\circ}$ and smaller than $270^{\circ}$, a relatively constant amount of neutrons is observed. The number of scattered neutrons also changes in this angular interval only by a slight amount. For $\vartheta$ equal to approximately $70^{\circ}$ or $290^{\circ}$ there is a very small amount of neutrons compared to the other directions, but almost $100 \%$ of them are deemed to be un-scattered (especially for distances $0.9 \mathrm{~m}$ and $2 \mathrm{~m}$ from the neutron source).

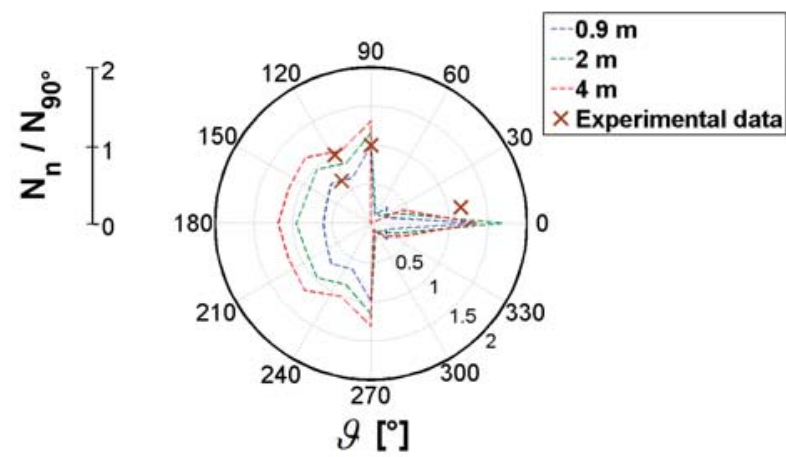

Fig. 5. The angular distribution of the amount of neutrons for the three distances from the neutron source. $\mathrm{N}_{90^{\circ}}$ represents the number of neutrons recorded at the closest surface located at $\vartheta=90^{\circ}$.

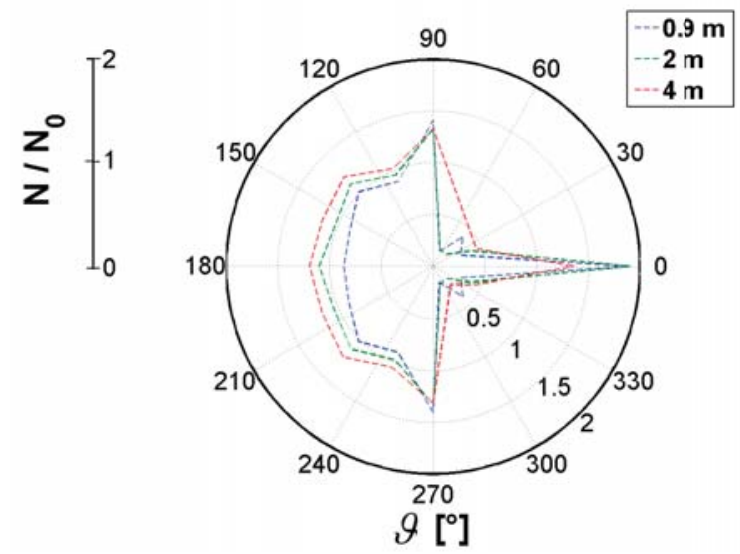

Fig. 6. The angular distribution of $\mathrm{N} / \mathrm{N}_{0}$ (neutron scattering) rate.

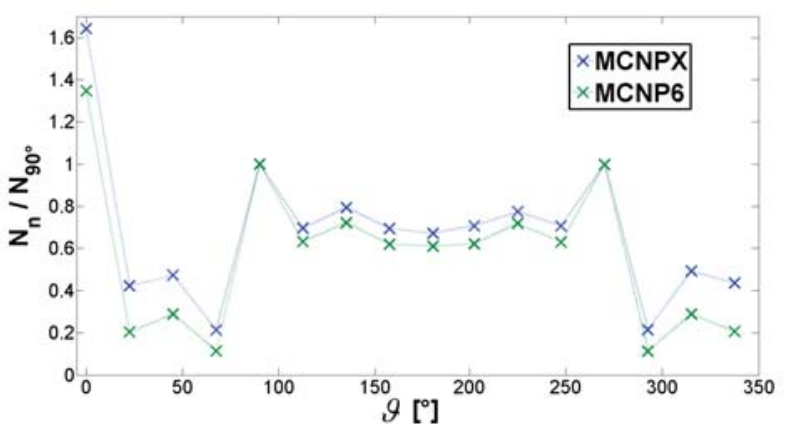

Fig. 7. MCNP6 and MCNPX results for the neutron emission anisotropy at $0.9 \mathrm{~m}$ from the neutron source. The obtained data represents the number of neutrons crossing the surface at $\vartheta=90^{\circ}$.

This information may be useful primarily for the time-of-flight (TOF) diagnostics which requires the number of scattered neutrons entering the detector be as small as possible.

\section{A comparison between the MCNPX and MCNP6 simulation results}

For the GIT-12 neutron anisotropy calculations the MCNPX code was also used and compared with the MCNP6 results (Fig. 7).

Both angular dependencies in Fig. 7 have a similar shape. In the angle interval from $70^{\circ}$ to $290^{\circ}$ they differ insignificantly, whereas in the remaining part of the graph the difference is more noticeable. Nevertheless, it is possible to regard the results as quite similar.

The differences between the two codes may be explained generally by the fact that the MCNP6 contains new physics, a different default behaviour and new or improved data compared to the MCNPX or the MCNP5. This means that particle trajectories computed in MCNPX may look different than trajectories generated by MCNP6, thus a different number of neutrons crossing the tally F1 surface may be obtained. The available overviews of the MCNP6, published by the LANL, consider the newer version of the code to be more reliable than the previous releases.

\section{Conclusions}

The credibility of the MCNP results for the GIT-12 device is supported by the similarity between the calculated waveform and the measured signal of the axial probe. It was found that the smallest amount of scattered neutrons is recorded at angles of approx. $70^{\circ}$ and $290^{\circ}$ with respect to the axial direction. At present the experimental data is too scarce to execute precise verification of calculations for neutron anisotropy, but the values measured so far roughly correspond to the MCNP simulation outcome. The MCNP6 and MCNPX calculations give comparatively similar results.

Acknowledgment. Supports by the grants MSMT no. LG13029, LH13283, GACR P205/12/0454, IAEA 
RC-16115, RC-16954, RC-16956, RC-17088 and SGS 13/194/OHK3/3T/13 are gratefully acknowledged.

\section{References}

1. Klir, D., Shishlov, A. V., Kokshenev, V. A., Kubes, P., Labetsky, A. Yu., Rezac, K., Cikhardt, J., Fursov, F. I., Kovalchuk, B. M., Kravarik, J., Kurmaev, N. E., Ratakhin, N. A., Sila, O., \& Stodulka, J. (2013). Characterization of neutron emission from mega-ampere deuterium gas puff Z-pinch at microsecond implosion times. Plasma Phys. Control. Fusion, 55(8), 085012. DOI: 10.1088/0741-3335/55/8/085012.

2. Rocca, J. J., Beethe, D. C., \& Marconi, M. C. (1988). Proposal for soft-X-Ray and XUV lasers in capillary discharges. Optics Lett., 13(7), 565-567. DOI: 10.1364/OL.13.000565.

3. Klir, D., Kubes, P., Rezac, K., Cikhardt, J., Kravarik, J., Sila, O., Shishlov, A. V., Kovalchuk, B. M., Ratakhin, N. A., Kokshenev, V. A., Labetsky, A. Yu., Cherdizov, R. K., Fursov, F. I., Kurmaev, N. E., Dudkin, G. N., Nechaev, B. A., Padalko, V. N., Orcikova, H., \& Turek, K. (2014). Efficient neutron production from a novel configuration of deuterium gas-puff Z-pinch. Phys.
Rev. Lett., 112 (9), 095001. DOI: 10.1103/PhysRevLett.112.095001.

4. Klir, D., Shishlov, A. V., Kubes, P., Rezac, K., Fursov, F. I., Kokshenev, V. A., Kovalchuk, B. M., Kravarik, J., Kurmaev, N. E., Labetsky, A. Yu., \& Ratakhin, N. A. (2012). Deuterium gas puff Z-pinch at currents of 2 to 3 mega-ampere. Phys. Plasmas, 19(3), 032706(11pp.). DOI: 10.1063/1.3696859.

5. Shishlov, A. V., \& Baksht, R. B. (2006). Experiments with a gas-puff-on-wire-array load on the GIT-12 generator for AlK-shell radiation production at microsecond implosion times. Dense Z-Pinches, 808(1). DOI: $10.1063 / 1.2159312$

6. Sila, O., Kubeš, P., Kravárik, J., Rezác, K., Klír, D., \& Cikhardt, J. (2013). Estimation of amount of scattered neutrons at devices PFZ and GIT-12 by MCNP simulations. Acta Polytechnica, 53 (2), 228-232.

7. Krasa, J., Kralik, M., Velyhan, A., Šolc, J., Juha, L., Scholz, M., Bienkowska, B., Ivanova-Stanik, I. M., Karpinski, L., Miklaszewski, R., Paduch, M., Schmidt, H., Tomaszewski, K., Klír, D., Kravárik, J., Kubeš, P., \& Rezáč, K. (2008). Anisotropy of the emission of DD-fusion neutrons caused by the plasma-focus vessel. Plasma Phys. Control. Fusion, 50(12), 125006. DOI: $10.1088 / 0741-3335 / 50 / 12 / 125006$. 